\title{
The enigmatic lack of glucose utilization in Streptomyces clavuligerus is due to inefficient expression of the glucose permease gene
}

\author{
Correspondence \\ Paloma Liras \\ paloma.liras@unileon.es
}

Received 26 October 2009

Revised 19 January 2010

Accepted 26 January 2010

\section{Rosario Pérez-Redondo, ${ }^{1,2}$ Irene Santamarta, ${ }^{1,2}$ Roel Bovenberg, ${ }^{3}$} Juan F. Martín ${ }^{1,2}$ and Paloma Liras ${ }^{1,2}$

\author{
${ }^{1}$ Área de Microbiología, Facultad de Ciencias Biológicas y Ambientales, Universidad de León, \\ 24071 León, Spain \\ ${ }^{2}$ Instituto de Biotecnología, INBIOTEC, Parque Científico de León, Avda. Real n¹, 24006 León, \\ Spain \\ ${ }^{3}$ DSM Anti-Infectives B.V., Delft, The Netherlands
}

\begin{abstract}
Streptomyces clavuligerus ATCC 27064 is unable to use glucose but has genes for a glucose permease $(g / c P)$ and a glucose kinase $(g / k A)$. Transformation of S. clavuligerus 27064 with the Streptomyces coelicolor g/cP1 gene with its own promoter results in a strain able to grow on glucose. The glcP gene of $S$. clavuligerus encodes a 475 amino acid glucose permease with 12 transmembrane segments. GlcP is a functional protein when expressed from the $S$. coelicolor g/cP1 promoter and complements two different glucose transport-negative Escherichia coli mutants. Transcription studies indicate that the $g / c P$ promoter is very weak and does not allow growth on glucose. These results suggest that $S$. clavuligerus initially contained a functional glucose permease gene, like most other Streptomyces species, and lost the expression of this gene by adaptation to glucose-poor habitats.
\end{abstract}

\section{INTRODUCTION}

A total of 53 putative carbohydrate uptake genes have been detected in the genome of the model organism Streptomyces coelicolor. ATP-binding cassette (ABC) transport systems account for the transport of ribose, lactose, maltose, xylose and sugar alcohols (Bertram et al., 2004). Glucose is transported by an $\mathrm{H}^{+}$-glucose symporter (van Wezel et al., 2005), and comparative analysis of gene sequences suggests that galactose is transported by an $\mathrm{Na}^{+}$-galactose symporter of the SSS (sodium solute symporters) family, similar to that found in Vibrio parahaemolyticus (Bertram et al., 2004). Fructose is incorporated by the phosphotransferase (PTS) system, as shown by the disruption of the ptsH gene (Nothaft et al., 2003b). A protein, GlpF, of the major intrinsic protein family appears to be responsible for glycerol-facilitated diffusion (Bertram et al., 2004; Hindle \& Smith, 1994). Recently, it has been shown that $\mathrm{N}$-acetyl-D-glucosamine (NAG) plays an important role in differentiation and antibiotic production in S. coelicolor (Rigali et al., 2006, 2008), in which this carbon source is transported by the sugar PTS system (Nothaft et al., 2003a).

Abbreviations: ABC, ATP-binding cassette; PTS, phosphotransferase.

The GenBank/EMBL/DDBJ accession numbers for the sequences of the regions upstream and downstream of $S$. clavuligerus glkA and g/cP are FN377746 and FN377747.
The behaviour of Streptomyces clavuligerus, the producer of clavulanic acid and cephamycin $\mathrm{C}$, in relation to carbon sources utilization is peculiar. This species is unable to grow on glucose as the sole carbon source; moreover, it does not use hexoses such as mannose, galactose or fructose, pentoses such as xylose, sugar alcohols such as mannitol or sorbitol, or disaccharides such as lactose or sucrose. Only glycerol, maltose and starch are efficiently used as carbon sources by S. clavuligerus (García-Domínguez et al., 1989).

If the systems for carbohydrate transport are conserved in different Streptomyces species, S. clavuligerus would be defective in: (i) ABC systems for lactose, xylose or mannitol uptake; (ii) the PTS system for fructose utilization; and (iii) the symporters required for glucose and galactose incorporation. This is an enigmatic situation for a soil-dwelling actinomycete.

One gene for glucose transport (araE, SAV2657) is present in the Streptomyces avermitilis genome, and two genes, glcP1 (SCO5578) and $g l c P 2$ (SCO7153), with 99.9\% nucleotide identity to each other, separated by $1.8 \mathrm{Mb}$ in the chromosome, occur in the S. coelicolor genome. They encode $100 \%$ identical GlcP1 and GlcP2 proteins. Both genes are expressed in glucose-grown cells of $S$. coelicolor, as shown by RT-PCR experiments; $g l c P 1$ is inducible by glucose while $g l c P 2$ transcripts are almost undetectable (van Wezel et al., 2005). In this work we report the effect of 
Table 1. Plasmids and strains used in this work

\begin{tabular}{|c|c|c|}
\hline Plasmid or strain & Relevant characteristics & Source or reference \\
\hline \multicolumn{3}{|l|}{ Plasmids } \\
\hline pFT256 & Contains $g l k A$ (SCO2126) controlled by its own promoter & $\begin{array}{l}\text { F. Titgemeyer, Friedrich-Alexander- } \\
\text { Universität, Erlangen, Germany }\end{array}$ \\
\hline pFT76 & E. coli vector containing $g l c P 2$ controlled by the $l a c Z$ promoter & van Wezel et al. (2005) \\
\hline pBluescript II SK $(+)$ & General cloning E. coli vector & Stratagene \\
\hline pUZ8002 & Conjugation helper plasmid & Paget et al. (1999) \\
\hline pLUXAR & E. coli/Streptomyces bifunctional conjugative-integrative vector & Rodríguez-García et al. (2007) \\
\hline pIJ699 & E. coli/Streptomyces bifunctional vector & Kieser et al. (2000) \\
\hline pIJ773 & pBluescript II SK $(+)$ containing an aac3(IV)-oriT cassette & Gust et al. (2003) \\
\hline pRA & E. coli/Streptomyces bifunctional conjugative-integrative vector & This work \\
\hline pRA-AB & $\begin{array}{l}\text { pRA containing PCR fragments assembled with the promoter region } \\
\text { of } S \text {. coelicolor } g l c P 1 \text { and the coding region of } S \text {. coelicolor } g l c P 1\end{array}$ & This work \\
\hline pRA-AD & $\begin{array}{l}\text { pRA containing PCR fragments assembled with the promoter region } \\
\text { of } S \text {. coelicolor glcP1 and the coding region of } S \text {. clavuligerus } g l c P\end{array}$ & This work \\
\hline pRA-CB & $\begin{array}{l}\text { pRA containing PCR fragments assembled with the promoter region } \\
\text { of } S \text {. clavuligerus } g l c P \text { and the } g l c P 1 \text { gene of } S \text {. coelicolor }\end{array}$ & This work \\
\hline pRA-CD & $\begin{array}{l}\text { pRA containing PCR fragments assembled with the promoter region } \\
\text { of } S \text {. clavuligerus } g l c P \text { and the } g l c P \text { gene of } S \text {. clavuligerus }\end{array}$ & This work \\
\hline pRA-AB-K & pRA-AB containing the glkA gene from pTF256 & This work \\
\hline pRA-1 & pRA containing the $S$. clavuligerus putative glkA activator & This work \\
\hline pRA-2 & $\begin{array}{l}\text { pRA containing the } S \text {. clavuligerus putative glkA activator and } \\
\text { upstream region }\end{array}$ & This work \\
\hline pAMB1 & E. coli/Streptomyces bifunctional vector, conjugative, replicative & This work \\
\hline pAMB1-1 & pAMB1-derived. Contains the $S$. clavuligerus putative glkA activator & This work \\
\hline pAMB1-2 & $\begin{array}{l}\text { pAMB1-derived. Contains the S. clavuligerus glkA activator and } \\
\text { upstream gene region }\end{array}$ & This work \\
\hline pBRel & $\begin{array}{l}\text { pFT76 vector lacking a } 1.2 \mathrm{~kb} B a m \mathrm{HI} \text { fragment with most of } \\
\text { the } g l c P 2 \text { gene }\end{array}$ & This work \\
\hline pKS-CD & $\begin{array}{l}\text { pBluescript KS II }(+) \text { containing PCR fragments assembled } \\
\text { with both promoter region and glcP gene of } S \text {. clavuligerus }\end{array}$ & This work \\
\hline $\mathrm{pglcP}$ & $\begin{array}{l}\text { E. coli vector containing } S \text {. clavuligerus glcP controlled by the } \\
\text { lacZ promoter }\end{array}$ & This work \\
\hline \multicolumn{3}{|c|}{ 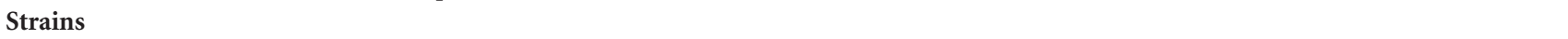 } \\
\hline E. coli DH5- $\alpha$ & General host strain & Hanahan (1983) \\
\hline E. coli ET12567 & Host strain for plasmid conjugation & MacNeil et al. (1992) \\
\hline E. coli $\mathrm{LM} 1$ & Glucose transport-negative mutant & Lengeler et al. (1981) \\
\hline E. coli LM1[pBRel] & E. coli LM1 transformant carrying the empty control vector $\mathrm{pBRel}$ & This work \\
\hline E. coli $\mathrm{LM} 1[\mathrm{pFT} 76]$ & $\begin{array}{l}\text { E. coli LM1 transformant carrying in pFT76 the } g l c P 2 \text { gene } \\
\text { controlled by the lacZ promoter }\end{array}$ & This work \\
\hline E. coli $\mathrm{LM} 1[\mathrm{p} g l c P]$ & $\begin{array}{l}\text { E. coli LM1 transformant carrying in pglcP the } S \text {. clavuligerus } \\
\text { glcP gene controlled by the lacZ promoter }\end{array}$ & This work \\
\hline E. coli LR2-175 & Glucose transport-negative mutant & Aulkemeyer et al. (1991) \\
\hline E. coli LR2-175[pBRel] & $\begin{array}{l}\text { E. coli LR2-175 transformant carrying the empty control } \\
\text { vector pBRel }\end{array}$ & This work \\
\hline E. coli LR2-175[pFT76] & $\begin{array}{l}\text { E. coli LR2-175 transformant carrying in pFT76 the } g l c P 2 \text { gene } \\
\text { controlled by the lacZ promoter }\end{array}$ & This work \\
\hline E. coli LR2-175[pglcP] & $\begin{array}{l}\text { E. coli LR2-175 transformant carrying in pglcP the } S \text {. clavuligerus } \\
\text { glcP gene controlled by the lac } Z \text { promoter }\end{array}$ & This work \\
\hline S. clavuligerus ATCC 27064 & Wild-type strain & ATCC \\
\hline S. coelicolor M145 & Prototroph & Kieser et al. (2000) \\
\hline S. clavuligerus::pRA & $\begin{array}{l}\text { Integrative exconjugant of } S \text {. clavuligerus } 27064 \text { carrying the } \\
\text { empty vector pRA }\end{array}$ & This work \\
\hline S. clavuligerus:: $\mathrm{pRA}-\mathrm{AB}$ & $\begin{array}{l}\text { Integrative exconjugant of } S \text {. clavuligerus } 27064 \text { carrying pRA } \\
\text { containing the } g l c P 1 \text { gene of } S \text {. coelicolor expressed from its own } \\
\text { promoter }\end{array}$ & This work \\
\hline
\end{tabular}


Table 1. cont.

\begin{tabular}{|c|c|c|}
\hline Plasmid or strain & Relevant characteristics & Source or reference \\
\hline S. clavuligerus: :pRA-AD & $\begin{array}{l}\text { Integrative exconjugant of } S \text {. clavuligerus } 27064 \text { carrying pRA } \\
\text { containing the } g l c P \text { gene of } S \text {. clavuligerus expressed from the } \\
g l c P 1 \text { promoter of } S \text {. coelicolor }\end{array}$ & This work \\
\hline S. clavuligerus::pRA-CD & $\begin{array}{l}\text { Integrative exconjugant of } S \text {. clavuligerus } 27064 \text { carrying pRA } \\
\text { containing the } g l c P \text { gene of } S \text {. clavuligerus expressed from its own } \\
\text { promoter }\end{array}$ & This work \\
\hline S. clavuligerus::pRA-AB-K & $\begin{array}{l}\text { Integrative exconjugant of } S \text {. clavuligerus } 27064 \text { carrying pRA } \\
\text { containing the } g l c P 1 \text { and } g l k A \text { genes of } S \text {. coelicolor expressed from } \\
\text { their own promoters }\end{array}$ & This work \\
\hline
\end{tabular}

S. coelicolor glcP1 (for glucose transport) and $g l k A$ (encoding the glucose kinase) genes on glucose utilization by $S$. clavuligerus. Using a combination of the S. coelicolor and $S$. clavuligerus glucose permease promoters coupled to either the promoterless $g l c P$ gene of $S$. clavuligerus or the promoterless $g l c P 1$ gene of $S$. coelicolor we conclude that expression of $\mathrm{glcP}$ from the native $S$. clavuligerus promoter is limiting for glucose utilization.

\section{METHODS}

Bacterial strains, plasmids and culture conditions. The bacterial strains and plasmids used in this study are listed in Table 1. Cultures of plasmid-bearing cells were supplemented with ampicillin $(50 \mu \mathrm{g}$ $\left.\mathrm{ml}^{-1}\right)$, chloramphenicol $\left(25 \mu \mathrm{g} \mathrm{ml}^{-1}\right)$, kanamycin $\left(25 \mu \mathrm{g} \mathrm{ml}^{-1}\right)$ or apramycin $\left(50 \mu \mathrm{g} \mathrm{ml}^{-1}\right)$, as appropriate.

Glucose transport-deficient Escherichia coli mutants and transformants carrying the $g l c P$ gene were grown on MacConkey or in VogesProskauer medium (Difco).

To inoculate cultures of $S$. coelicolor, spores were germinated in $2 \times$ tryptone yeast extract (TY) medium (Kieser et al., 2000). $S$. clavuligerus cultures were inoculated using culture aliquots [grown in tryptone soya broth (TSB) medium] kept frozen in $20 \%(\mathrm{v} / \mathrm{v})$ glycerol. Streptomyces strains were grown in $500 \mathrm{ml}$ baffled flasks at $28{ }^{\circ} \mathrm{C}$ and 220 r.p.m.

To check the growth on different carbon sources, strains were inoculated in $50 \mathrm{ml}$ of inoculum medium (IM), which contains basal medium (BM) supplemented with $0.2 \%$ L-asparagine, $1 \%$ glycerol, $0.1 \% \mathrm{NH}_{4} \mathrm{Cl}$ and $0.1 \%$ yeast extract (Aharonowitz \& Demain, 1978). After $24 \mathrm{~h}$, the mycelium was washed and different volumes, as appropriate, were transferred to $50 \mathrm{ml} \mathrm{BM}$ containing $0.1 \% \mathrm{NH}_{4} \mathrm{Cl}$ and $1 \%$ of the carbon source tested. Glucose solutions were treated with $\alpha$-glucosidase to eliminate traces of maltose present in commercial glucose supplies. For the growth of S. coelicolor, BM was supplemented with the trace elements described by Chatterjee \& Vining (1981).

Glucose in the culture supernatants was quantified with the Glucose Assay kit (Sigma).

Growth was determined by measuring total DNA by the diphenylamine method (Burton, 1968).

DNA manipulations. Plasmid DNA isolation, restriction endonuclease digestions, ligations and transformation of $E$. coli were performed using standard techniques (Sambrook et al., 1989). Genomic DNA preparations from Streptomyces were carried out according to Kieser et al. (2000). Non-radioactive hybridizations were performed following the protocol given in the DIG System kit (Roche), and detection was done using 5-bromo-4-chloro-3-indolyl phosphate and nitro blue tetrazolium.

DNA sequencing. DNA sequencing of the PCR-amplified fragments was performed using a MegaBACE 500 DNA Analysis System (Amersham Biosciences) sequencer. Subclones were sequenced from universal primers, checking the information on both strands. The sequences of the regions upstream and downstream of $S$. clavuligerus $g l k A$ and $g l c P$ were provided by DSM (Delft, The Netherlands) and have been deposited in the EMBL database with the accession numbers FN377746 and FN377747.

RNA isolation. RNAprotect Bacteria reagent (Qiagen) was used for stabilization of collected samples for RNA extraction. Mycelium was treated with lysozyme $\left(30 \mathrm{mg} \mathrm{ml}^{-1}\right)$ and RNeasy Mini Spin Columns (Qiagen) were used for RNA isolation according to the manufacturer's instructions, including a phenol extraction step before transferring the lysate to the column. RNA preparations were incubated with Turbo DNase (Ambion) to eliminate any chromosomal DNA contamination. RNA quantification was done with a NanoDrop ND-1000 UV-vis spectrophotometer.

PCR and non-quantitative RT-PCR analysis. Oligonucleotide primers used in this work are shown in Table 2. All PCRs were performed in a TGradient (Biometra) thermocycler using Platinum Pfx DNA polymerase (Invitrogen). The dNTP mix was prepared from individual nucleotides using a ratio of $15 \mathrm{~A}: 15 \mathrm{~T}: 35 \mathrm{G}: 35 \mathrm{C}$ to improve amplification of high-G + C DNA content. Total DNA from S. coelicolor was used to amplify the glcP1 (SCO5578) promoter with primers CRP1/CRP2 and the glcP1-coding region with CRP3/CRP4, whereas $S$. clavuligerus total DNA was used to amplify the $g l c P$ promoter using the oligonucleotide pair CRP5/CRP6 and the glcP-coding region with CRP7/CRP8. PCR products were subcloned into pBluescript II $\mathrm{SK}(+)$ and sequenced for confirmation before assembling the promoter-gene constructs for integration in Streptomyces.

Gene expression analysis by RT-PCR was done with the SuperScript One-Step system (Invitrogen) using 150-200 ng total RNA as template. Primers were designed to generate PCR products of approximately 500-600 bp. RT-PCR conditions were as follows: first strand cDNA synthesis, $50{ }^{\circ} \mathrm{C}$ for $30 \mathrm{~min}$ followed by $94{ }^{\circ} \mathrm{C}$ for 2 min; PCR amplification was performed in two phases: three cycles of $94{ }^{\circ} \mathrm{C}$ for $30 \mathrm{~s}, 68-62{ }^{\circ} \mathrm{C}$ (depending on the set of primers used) 
Table 2. Oligonucleotides used in this work

\begin{tabular}{|c|c|c|c|}
\hline Primer & Sequence $\left(5^{\prime} \rightarrow 3^{\prime}\right)^{*}$ & Restriction site & Use \\
\hline GLCP1 $1 \dagger$ & GCGATGGGCGGTTTCCTG & & Forward primer for $S$ coelicolor glcP1 gene RT-PCR \\
\hline GLCP $2 \dagger$ & CGGGGATGGCGAAGGACAG & & Reverse primer for $S$. coelicolor glcP1 gene RT-PCR \\
\hline GLCP5 & GGATCGACCGGGCCAGGAAGG & & Forward primer for S. clavuligerus glcP gene RT-PCR \\
\hline GLCP6 & CCCCGAGGAGCACCCAGACCA & & Reverse primer for $S$. clavuligerus glcP gene RT-PCR \\
\hline RT-glk7 & CCGCGGGCTATGTGGACGAC & & Forward primer for S. clavuligerus glkA gene RT-PCR \\
\hline RT-glk8 & GAGACGCCGCCGCCGATGATGAAC & & Reverse primer for S. clavuligerus glkA gene RT-PCR \\
\hline CRP1 & GAATTCGTTCTTCAAGACTTGAAC & EcoRI & Forward primer for S. coelicolor glcP1 promoter cloning \\
\hline CRP2 & CATATGTACTCCTCGGGCTAC & NdeI & Reverse primer for S. coelicolor glcP1 promoter cloning \\
\hline CRP3 & 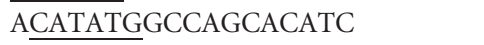 & NdeI & Forward primer for S. coelicolor glcP1 gene cloning \\
\hline CRP4 & AGATCTGCGGGTCAGCCCATTTC & $B g l \mathrm{II}$ & Reverse primer for S. coelicolor glcP1 gene cloning \\
\hline CRP5 & 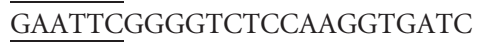 & EcoRI & Forward primer for $S$. clavuligerus $g l c P$ promoter cloning \\
\hline CRP6 & CATATGAACTCCTGGGGCCG & NdeI & Reverse primer for $S$. clavuligerus glcP promoter cloning \\
\hline CRP7 & 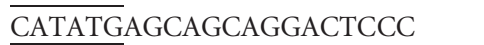 & NdeI & Forward primer for S. clavuligerus glcP gene cloning \\
\hline CRP8 & 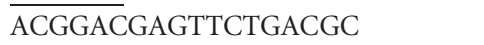 & & Reverse primer for $S$. clavuligerus glcP gene cloning \\
\hline
\end{tabular}

${ }^{\star}$ Restriction sites added in the $5^{\prime}$ region of cloning oligonucleotides are underlined.

$\dagger$ Primers GLCP1/GLCP2 are specific for $g l c P 1$ and do not amplify the $g l c P$ gene.

$\ddagger$ Primers GLCP5/GLCP6 are specific for $g l c P$ and do not amplify the $g l c P 1$ gene.

for $30 \mathrm{~s}, 72{ }^{\circ} \mathrm{C}$ for $40 \mathrm{~s}$ and $20-35$ cycles of $94{ }^{\circ} \mathrm{C}$ for $30 \mathrm{~s}, 66-58{ }^{\circ} \mathrm{C}$ (depending on the set of primers) for $30 \mathrm{~s}$ and $72{ }^{\circ} \mathrm{C}$ for $40 \mathrm{~s}$. Systematically, to compare non-saturated amplification products, a decreasing number of PCR cycles were performed. Negative controls were carried out with each set of primers and Platinum Taq DNA polymerase (Invitrogen) to verify the absence of contaminating DNA in the RNA preparations.

Glucose kinase assays. S. clavuligerus was grown in TSB medium [Trypticase $\left(30 \mathrm{~g} \mathrm{l}^{-1}\right)$ in soy broth] and $S$. coelicolor in a mixture of TSB and yeast extract/malt extract (YEME) media (40:60\%) (Kieser et al., 2000) for $24 \mathrm{~h}$, and the mycelium was centrifuged and transferred to starch and asparagine (SA) medium (Aidoo et al., 1994) supplemented with glucose $(1 \%)$ under vigorous shaking for $10 \mathrm{~h}$. Cells were harvested and washed twice, and $1 \mathrm{~g}$ mycelium was

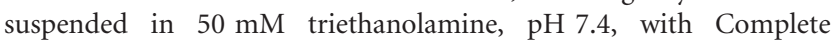
Protease Inhibitor Cocktail (Roche). Mechanical disruption was performed with a FastPrep system (Thermo Scientific), cell debris was removed by centrifugation and the supernatant was filtered through a PD-10 desalting column (GE Healthcare).

Glucose kinase activity was measured spectrophotometrically by monitoring the formation of NADPH in a glucose-6-phosphate dehydrogenase coupled reaction, as described by García-Domínguez et al. (1989). Enzyme activity is expressed as units, where one unit is the amount of enzyme that produces $1 \mathrm{nmol} \mathrm{NADPH} \min ^{-1}$.

Construction of plasmids. pRA is an EcoRV-digested pLUXAR recircularized. pAMB-1 is pIJ773 carrying a $4.9 \mathrm{~kb}$ HindIII fragment from pIJ699.

The $S$. clavuligerus putative $g l k A$ activator and the ORF upstream were subcloned in a $2096 \mathrm{bp} N$ siI-BclI fragment in the single-copy integrative pRA plasmid digested with EcoRV, to give pRA-2. From pRA-2 a 926 bp XmnI-EcoRI fragment containing only the putative glkA activator was rescued and subcloned in EcoRV/EcoRI-digested pRA to obtain pRA-1. The same 2096 and 926 bp DNA fragments were subcloned in the multicopy plasmid pAMB1 linearized with EcoRV to get pAMB1-2 and pAMB1-1.

The plasmids harbouring PCR fragments with S. clavuligerus or $S$. coelicolor glucose permease promoters or coding regions were used to assemble different arrangements of promoter and gene by means of the restriction sites included in the oligonucleotides designed. These fragments were then subcloned in EcoRI/BamHI-digested pRA to give: (i) pRA-AB (A corresponds to the promoter and $\mathrm{B}$ to the ORF in S. coelicolor), in which in a $1617 \mathrm{bp}$ fragment the S. coelicolor $g l c P 1$ promoter controls the S. coelicolor glcP1 gene; (ii) pRA-AD, in which in a $1685 \mathrm{bp}$ fragment the $S$. coelicolor glcP1 promoter (A) controls the $S$. clavuligerus glcP gene (D); (iii) pRA-CB, in which in a $1633 \mathrm{bp}$ fragment the $S$. clavuligerus $g l c P$ promoter (C) controls the $S$. coelicolor glcP1 gene (B); and (iv) pRA-CD, in which in a 1701 bp fragment the $S$. clavuligerus $g l c P$ promoter (C) controls the S. clavuligerus glcP gene (D) (see Fig. 5).

To obtain pRA-AB-K, a $1 \mathrm{~kb}$ DNA fragment isolated from pFT256 carrying the $S$. coelicolor glkA gene expressed from its own promoter, was ligated into the EcoRV site of pRA-AB.

Plasmid DNA was introduced into Streptomyces by conjugation using E. coli ET12567[pUZ8002] as the donor strain. Transconjugants were isolated on mannitol-soya medium (Kieser et al., 2000) and selected with apramycin. The integration of pRA-derived plasmids or the presence of the autonomous pAMB1-derived plasmids was checked by total DNA hybridization or by plasmid recovery.

Plasmid pglcP was constructed to check the functionality of S. clavuligerus glucose permease. A $1.5 \mathrm{~kb}$ SacI-Xbal fragment containing the $g l c P$-coding region and $25 \mathrm{nt}$ upstream was obtained from pKS-CD and ligated downstream of the lacZ promoter in SacI/ HindIII-digested pFT76. The control plasmid pBRel was constructed by deletion of a $1.2 \mathrm{~kb}$ internal fragment of glcP2 in BamHI-digested pFT76.

\section{RESULTS}

\section{S. clavuligerus contains a glkA gene and has normal glucose kinase activity}

To understand the lack of glucose utilization by S. clavuligerus we searched for a glucose kinase gene $(g l k A)$ in this organism. An S. clavuligerus glkA gene was detected 
by hybridization of digested total DNA with a $1 \mathrm{~kb}$ probe containing the S. coelicolor glkA gene (Angell et al., 1992). The sequence of a hybridization-positive $4.4 \mathrm{~kb} B s t$ EII DNA fragment revealed a gene organization similar to that of S. coelicolor, S. avermitilis and Streptomyces griseus (Fig. 1a). The $g l k A$ gene encodes a 313 amino acid protein $\left(M_{\mathrm{r}} 32273\right.$, deduced pI 5.8) of the ROK family, $81 \%$ identical in amino acid sequence to the $S$. coelicolor homologue (Fig. 1b).

The glkA gene is located downstream of a gene (orf2) encoding an ion transporter ATPase, and of a gene (orf3) for a putative glucose kinase activator (with $57.6 \%$ identity to SCO2127) (Guzmán et al., 2005). Downstream of glkA and separated by $679 \mathrm{nt}$ is a gene (orf5) encoding a product of unknown function.
The in vitro glucose kinase activity of S. clavuligerus was determined in three different experiments and compared with that of S. coelicolor. Both strains were grown in SA medium supplemented with $1 \%$ glucose. A specific activity of $23.2 \pm 3.2 \mathrm{mU}$ (mg protein) ${ }^{-1}$ was found in S. coelicolor cell extracts, while the mean specific glucose kinase activity in S. clavuligerus was $17.25 \pm 2.3 \mathrm{mU}(\mathrm{mg} \text { protein })^{-1}$.

Transformant S. clavuligerus [pFT256] carrying the $S$. coelicolor glkA gene expressed from its own promoter was also not able to use glucose, although the glucose kinase activity in the transformant was $19.7 \pm 1.6 \mathrm{mU}(\mathrm{mg}$ protein $)^{-1}$.

The SCO2127 gene of S. coelicolor has a positive effect on glucose uptake and glucose kinase activities (Guzmán et al., 2005), possibly due to transcription activation. Therefore,

(a)

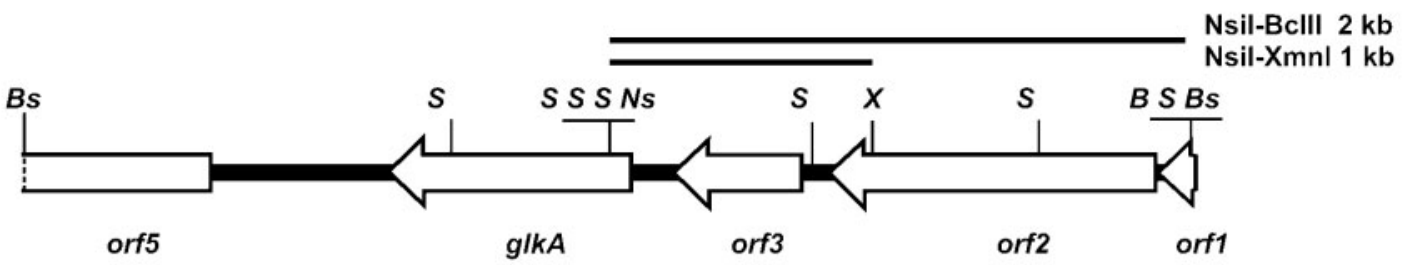

(b)

MGLTIGVDIGGTKIAAGVVDEEGNILSTHKVPTPTTPEAIVDAIASAVEGARVGHEIVAVGIGAAGYVNRQRSTVYFAPNIDWRQ 85 MGLTIGVDIGGTKIAAGVVDEAGAIIETHTVATPSTPGGIVDAICSAVAGAGEGHRIEAVGIGAAGYVDDKRATVLFAPNIDWRH 85

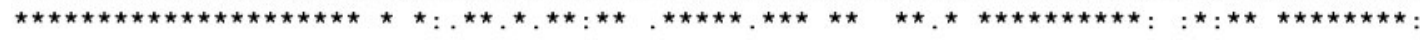

EPLKEKVEARVGLPVVVENDANAAAWGEYKFGGGKGHRNVICITLGTGLGGGIIIGNKLRRGHFGVAAEFGHIRMVPDGLLCGCG 170 EPLKDKVEQRVGLPVVVENDANAAAWGEFRFGAGQGHEDVIVITLGTGLGGGIIIGNKLRRGRFGVAAEFGHIRVVPDGLLCGCG 170

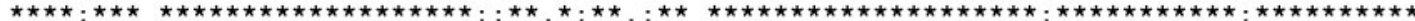

SQGCWEQYASGRALVRYAKQRANATPERAEVLLALGDGTPDGIEGKHISVAARQGCPVAVDSYRELARWAGAGLADLASLFDPSA 255 SQGCWEQYASGRALVRYARQRAAAAPEAAAVLLGLGDGTPEGIEGRHVSEAARQGDPVAVDSFRELARWAGAGLADLASLFDPSA 255

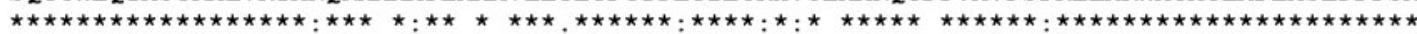
FIVGGGLSDEGDLVLDPIRKSYKRWLVGGNWRPVADVIAAOLGNKAGLVGAADIAREPDPIM 317 S. coelicolor GIkA FIIGGGVSDEGELVLEPIRKSFRRWLIGAQWRPHAQVVAARLGNRAGLVGAADLARQG $\quad 313$ S. clavuligerus GIkA

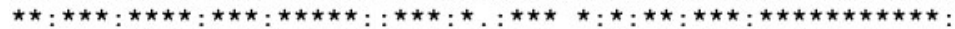

(c)
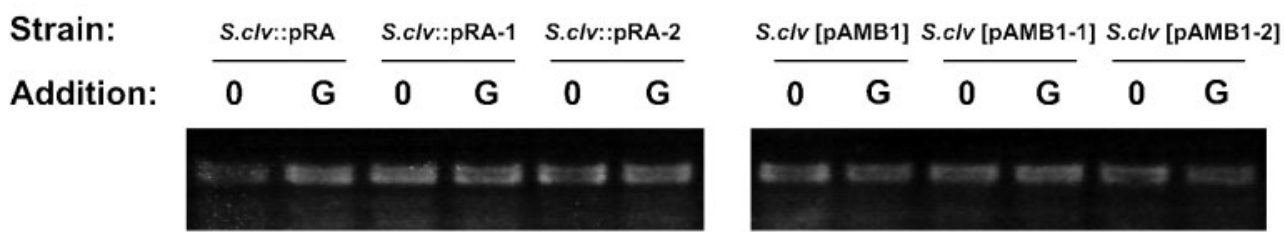

Fig. 1. Characteristics of the glucose kinase gene of S. clavuligerus. (a) Organization of a BstEll DNA fragment containing the incomplete orf1 (encoding a putative cyclase-dehydratase), orf2 (encoding an ion transport ATPase), orf3 (encoding a putative glkA activator), glkA (orf4) and an incomplete orf5 (encoding a protein of unknown function). The glkA gene is named SCCG_05538.1 in the S. clavuligerus Broad Institute Project Sequence. Bs, BstEll; S, Sall; Ns, Nsil; X, Xmnl; B, Bcll. Horizontal bars indicate the DNA fragments used in the construction of pRA-1, pRA-2, pAMB1-1 and pAMB1-2. (b) Comparison of $S$. clavuligerus and S. coelicolor GlkA. The characteristic set of cysteines required for activity (Mesak et al., 2004) is indicated with a horizontal line. Identical amino acids are indicated with asterisks. (c) Expression of glkA, detected by RT-PCR, in strains carrying different fragments containing orf3 in single-copy integrated form (S. clavuligerus::pRA-1, S. clavuligerus::pRA-2), in a multicopy plasmid (S. clavuligerus[pAMB1-1], S. clavuligerus[pAMB1-2]) and in negative controls (S. clavuligerus : : pRA, S. clavuligerus[pAMB1]). RNA was obtained in SA medium under control conditions (0) and $3 \mathrm{~h}(\mathrm{G})$ after glucose supplementation. 
S. clavuligerus strains carrying orf 3 , the SCO2127 homologous gene, in a mono-copy integrative plasmid (named pRA-1 and pRA-2), or in a multi-copy plasmid (named pAMB1-1 and pAMB1-2) along with their controls (pRA and pAMB1) were constructed. When the RNA of these strains, grown in SA medium or SA supplemented with glucose, was tested by RT-PCR, they showed the same amplification intensity for the glkA gene (Fig. 1c). This indicates that $S$. clavuligerus orf 3 is not a glkA activator and that glucose does not stimulate $g l k A$ transcription.

\section{The S. clavuligerus glcP gene encodes a glucose permease functional in $E$. coli}

Labelled glucose is barely incorporated in S. clavuligerus (García-Domínguez et al., 1989). To test whether a glucose permease gene exists in S. clavuligerus, total DNA was hybridized with a $1.4 \mathrm{~kb}$ DNA fragment containing the whole S. coelicolor glcP2 gene. A clear signal of $6 \mathrm{~kb}$ was found in BstEII-digested DNA; therefore, the genome of S. clavuligerus, available at DSM (Delft, The Netherlands), was searched for a gene homologous to $S$. coelicolor glcP1 or glcP2. One single glucose permease gene located downstream of a chromosome segregation gene (orf6) was found, while in the opposite orientation, genes for a putative acetyltransferase (orf8) and a putative docking protein $(f t s Y)$ were present (Fig. 2a).

The $S$. clavuligerus glucose permease gene was named $g l c P$ to differentiate it from the S. coelicolor $g l c P 1$ and glcP2 genes. It encodes a protein with $80-81 \%$ identity to $S$. coelicolor GlcP1, S. avermitilis AraE and S. griseus SGR1900 (Fig. 2b). The protein $\left(M_{\mathrm{r}} 50539\right.$, pI 8.4), contains 12 membrane-spanning segments as found in other $\mathrm{H}^{+}$ glucose symporters.

Two glucose transport-negative mutants, E. coli LM1 and E. coli LR2-175 (Aulkemeyer et al., 1991; Lengeler et al., 1981), were transformed with plasmid pglcP, in which S. clavuligerus $g l c P$ is expressed from the lac $Z$ promoter, and with plasmids pFT76 (containing S. coelicolor glcP1) and pBRel, as positive and negative controls, respectively. The strains were streaked on MacConkey agar and on MacConkey agar supplemented with $50 \mathrm{mM}$ glucose. Transformants E. coli LM1[pglcP] and E. coli LR2$175[\mathrm{p} g l c P]$ were able to use glucose, as indicated by the yellow halo formed around the patches (Fig. 3a), whereas the parental strains and transformants E. coli LM1[pBRel] and E. coli LR2-175[pBRel] did not produce yellow zones. In addition, the strains were grown for $48 \mathrm{~h}$ in VogesProskauer medium and supplemented with methyl red. The culture broth of E. coli LR2-175[pFT76] and E. coli LR2-175[p $g l c P]$ turned from slightly yellow to intense red (Fig. 3b), indicating acidification due to glucose fermentation, while the parental mutant and control negative strain retained the pale yellow colour, confirming that they are not able to use glucose. This indicates that S. clavuligerus glucose permease is functional when expressed in E. coli.

\section{glcP is weakly expressed in S. clavuligerus}

In $S$. coelicolor the essential glucose permease is $g l c P 1$, while the duplicate $g l c P 2$ gene can be deleted without a significant effect on glucose utilization. The two genes have substantial differences in their $5^{\prime}$-upstream regions. When compared with the homologous regions in $S$. avermitilis araE and $S$. clavuligerus $g l c P$, an overall similarity is found between $g l c P 1$ and araE upstream regions. The -10 (TAGTCT) and -35 (TTGACT) sequences of glcP1 (van Wezel et al., 2005) are perfectly conserved in araE. However, the homology is very low with the promoter regions of $S$. coelicolor glcP2 (results not shown) or S. clavuligerus $g l c P$, in which the -10 and -35 regions are difficult to recognize (see Fig. 2c).

The $g l c P$ gene in S. clavuligerus is $161 \mathrm{nt}$ downstream of orf6 and $55 \mathrm{nt}$ upstream, and in the opposite sense to orf8 (Fig. 2a). RT-PCR analysis was done using RNA isolated from S. clavuligerus::pRA (control strain carrying the integrated pRA empty plasmid) grown in: (i) BM plus glycerol, (ii) BM plus glucose (Fig. 4, upper panel) or (iii) SA, and (iv) SA plus glucose (Fig. 4, lower panel). To discriminate strand-specific transcription, the experiment was performed in two steps: (i) mRNA retrotranscription to obtain cDNA was done using primers GLCP5 or GLCP6 separately, and (ii) the second oligonucleotide (either GLCP6 or GLCP5, respectively) was added later for the PCR process. When the cDNA was obtained using GLCP6, complementary to the glcP mRNA (Fig. 4, lanes 3 and 7), the yield of amplified product was barely detectable and clearly lower than when the retrotranscription was done with the GLCP5 primer (Fig. 4, lanes 2 and 6). These results indicate that a very weak transcription of $g l c P$ occurs but that most of the signal in that region is due to an antisense transcript, probably originating in orf8. The residual expression of $g l c P$ is not affected by the presence of glucose in the cultures (Fig. 4, lanes 3 and 7).

\section{Construction of S. clavuligerus recombinant strains able to use glucose}

The promoter regions of $g l c P 1$ from $S$. coelicolor and $g l c P$ from S. clavuligerus, as well as S. coelicolor glcP1- and $S$. clavuligerus $g l c P$-coding sequences, were amplified by PCR. The four PCR products were assembled in the four combinations (two promoters $\times$ two ORFs) indicated in Fig. 5a and subcloned in the integrative single-copy plasmid pRA in such a way that each gene was expressed either from its own promoter (constructions pRA-AB and pRA-CD, where the last two letters refer to the DNA fragments combined) or from the promoter of the homologous gene (constructions $\mathrm{pRA}-\mathrm{AD}$ and $\mathrm{pRA}-\mathrm{CB}$ ). $S$. clavuligerus exconjugants carrying the integrated plasmids were grown in BM medium with glucose or glycerol as sole carbon source. All the strains grew on glycerol but only S. clavuligerus::pRA-AB and S. clavuligerus::pRA$\mathrm{AD}$, expressing $S$. coelicolor glcP1 and S. clavuligerus glcP, respectively, from the $S$. coelicolor glcP1 promoter, grew on 
(a)

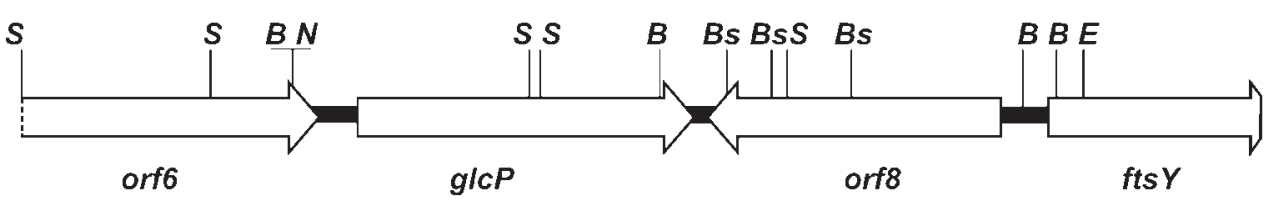

(b)

V-SSRTPPSPPPPGVPAAAHPE $\overline{\text { HLGHVVFITASAAMGGFLFGYDSVINGAVEAIRDRYAIGSAELAQVIAIALIGCAIGAATAG }} 84$ MASTSQAPSP---G-AGTAHPDHLGHVIFIAAAAAMGGFLFGYDSSVINGAVEAIRDRYDVGSAVLAQVIAVALIGCAIGAATAG 81

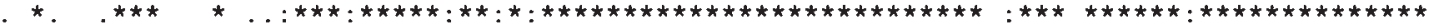

RIADRIGRIACMRIAALIFAASAVGSALPFALWDLAMWRVIGGFAIGMASVIGPAYIAEVSPPAYRGRLASFQQAAIVIGIAVSQ 169 RIADRIGRIRCMQIAAVLFTVSAVGSALPFALWDLAMWRIIGGFAIGMASVIGPAYIAEVSPPAYRGRLGSFQQAAIVIGIAVSQ 166

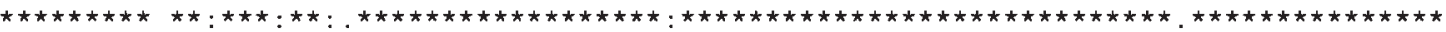

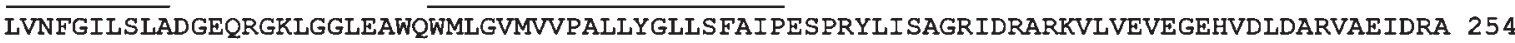
LVNWGLLNAAGGDQRGELMGLEAWQVMLGVMVIPAVLYGLLSFAIPESPRFLISVGKRERAKKIIEEVEGKDVDFDARVTEIEHA 251

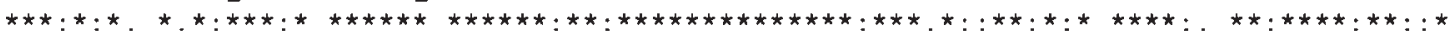

MRSEHRSTFRDLLGGRFGFIPIVWIGIGLSVFQQLVGINVVFYYSSTLWQSVGIDPTSSFFYSFTSSIVNIIGTVIAMVLVDRIG 336 MHREEKSSFKDLLGGSFFFKPIVWIGIGLSVFQQFVGINVAFYYSSTLWQSVGVDPADSFFYSFTTSIINIVGTVIAMIFVDRVG 333

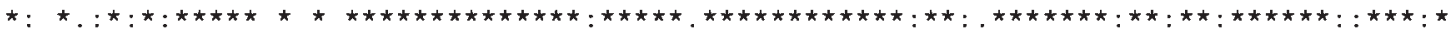

RRPLALIGSAGMAISLALEAWAF SAPLVNGKLPGTQGTVALIAAHVFVLFFALSWGVVVWVLLGEMFPNRIRAAALGVAASAQWL 421 RKPLALIGSVGMVIGLALEAWAF SFDLVDGKLPATQGWVALIAAHVFVLFFALSWGVVVWVFLGEMFPNRIRAAALGVAASAQWI 418

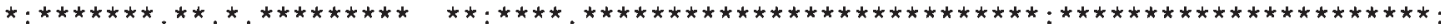

ANWLITATFPSLSDWNLSATYIIYASFAVLSIPFVHFFVKETKGKPLEEMG ANWAITASFPSLADWNLSGTYVIYTIFAALS I PFVLKFVKETKGKALEEMG

(c)

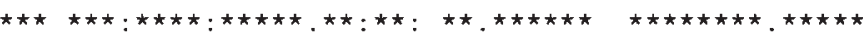

475 S. clavuligerus GlcP 472 S. coelicolor GlcP1

TGTCCATGGGTTGAGTTCAAGTCTTGAAGGTAAGTGCAACTCTGAGTCGTTAAAACTCACAGCGGACCCCCTATTGACTT TGAACCCTCGTT--CTTCAAGACTTGAACACATAACCACTTCAGCTCCCCTGAAAGTCACAGCTCTCGCCCTCTTGACTT TGAGCCCGCCCCGGACGGACCCGGGGTCA-GGCCGACGGCCC--AGCCCC

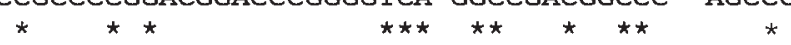

CGTTACTTGAAGGCATAGTCTCTGCAACGTTGCTTTTACCTTCAGGTGGTGGGCGGCGTGAAGTTGTGCGCCACTGGAAC

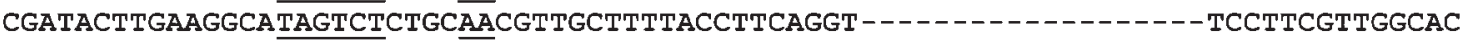
GGTGGTGGGCCGGGCCCGGCCGTGCGCCACTGGAGGGACCCGCTCCGC $-\ldots \ldots$

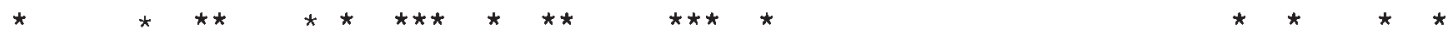

CATGACTGAAGGGCTCGCCCCCACCCCCGGCAGCGAGGCCGGTGGCCCGAGGAGTTT TACGTG araE $S$. avermitilis CT----CGAAGGGTGAACCGC----CCCGGCAGCGTTGCCGGTAGCCCGAGGAGTA-CACGTG glcP1 s. coelicolor CCCCGTGGGCGGGTGCGCCGCG---GACGGAGCTCCGGGCGCCGGCCCCAGGAGTTG---ATG glcP s. clavuligerus

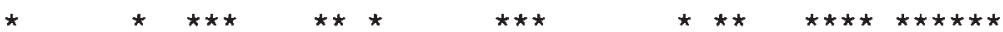

Fig. 2. Characteristics of the glucose permease gene $(g / c P)$ of $S$. clavuligerus. (a) Organization of the genome in the region around $g / c P$ : incomplete orf6 (encoding a chromosome segregation protein), glcP (orf7), orf8 (encoding an acetyltransferase) and incomplete $\mathrm{fts} Y$. The glcP gene is named SCCG_07234.1 in the S. clavuligerus Broad Institute Project Sequence. S, Sall; $B, B c l l ; N$, Ncol; Bs, BstEll; E, EcoRI. (b) Comparison of the proteins encoded by S. clavuligerus g/cP and S. coelicolor g/cP1. The 12 transmembrane motifs predicted for GlcP are indicated with horizontal lines. (c) Comparison of the promoter-containing intergenic regions upstream of araE from $S$. avermitilis, g/cP1 from $S$. coelicolor and glcP from S. clavuligerus. The -10 and -35 regions and the transcription start point of the S. coelicolor glcP1 promoter (van Wezel et al., 2005) and the putative homologous regions in the $S$. avermitilis araE promoter are underlined.

glucose (Fig. 5b). No growth was observed of S. clavuligerus::pRA-CD or of S. clavuligerus::pRA-CB, in which the promoter of the native $S$. clavuligerus $g l c P$ gene was used. This lack of growth confirms that the promoter of $g l c P$ is weak or non-functional.

The growth on glucose of $S$. clavuligerus::pRA-AB and S. clavuligerus::pRA-AD was delayed as compared with that on glycerol and the cellular DNA content was lower, suggesting a poor flow through the early steps of glucose metabolism in S. clavuligerus.

To improve the growth on glucose, the S. coelicolor glkA gene, expressed from its own promoter, was subcloned into plasmid pRA-AB to give plasmid pRA-AB-K, which therefore carries both $g l c P 1$ and $g l k A$ from $S$. coelicolor 
(a)

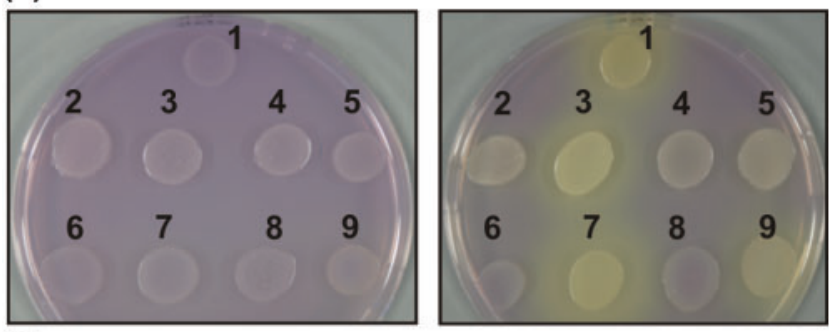

(b)

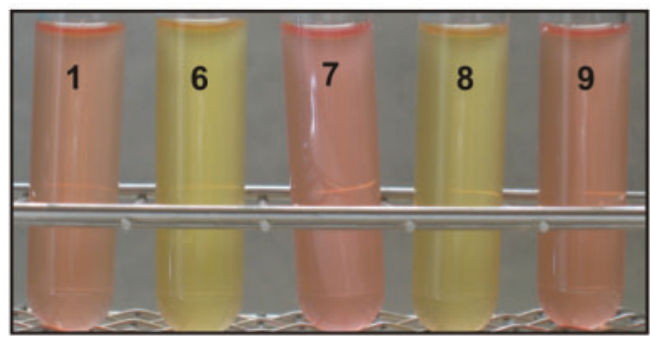

Fig. 3. Complementation of glucose transport-negative $E$. coli strains by $S$. clavuligerus g/cP. (a) MacConkey agar plates without (left) and supplemented with $50 \mathrm{mM}$ glucose (right). (b) Liquid Voges-Proskauer with methyl red. E. coli strains: (1) DH5- $\alpha,(2)$ LM1, (3) LM1[pFT76], (4) LM1[pBRel], (5) LM1[pg/cP], (6) LR2175, (7) LR2-175[pFT76], (8) LR2-175[pBRel], (9) LR2175[pg/cP].

(Fig. 6a). The good growth of S. clavuligerus::pRA-AB-K on glucose shows that the combination of glcP1 and glkA increases the growth of the culture (Fig. 6b, left panel).

Studies of glucose utilization by the exconjugants (Fig. 6b, right panel) indicate that $S$. clavuligerus::pRA (control
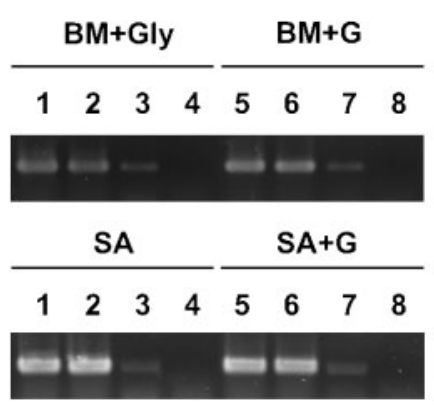

\section{glcP \\ S. clavuligerus::pRA}

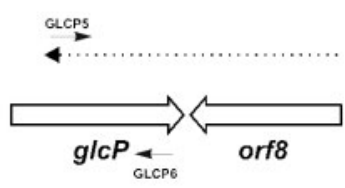

Fig. 4. Transcription of $S$. clavuligerus g/cP. Amplification of the S. clavuligerus::pRA g/cP gene from RNA obtained in BM medium supplemented with either glycerol $(B M+G l y)$ or glucose $(B M+G)$ (upper panel), or in SA medium and SA supplemented with glucose (SA+G) (lower panel). Lanes: 1 and 5, one-step RTPCR using oligonucleotides GLCP5 and GLCP6; 4 and 8, PCR negative control with the same oligonucleotides; 2 and 6, PCR performed using cDNA obtained with GLCP5 in the reversetranscription step (see scheme at the right side); 3 and 7, PCR performed using cDNA obtained with GLCP6 in the reversetranscription step. strain) did not consume glucose, but S. coelicolor (not shown) and $S$. clavuligerus exconjugants carrying one additional copy of the glucose permease genes consumed glucose to different degrees. Glucose depletion was faster in S. clavuligerus:: pRA-AB-K than in S. clavuligerus::pRA$\mathrm{AB}$ (Fig. 6b, right panel), reflecting the cooperative effect of the $g l c P 1$ and $g l k A$ genes, while S. clavuligerus::pRA-AD, expressing the native $g l c P$ gene from the $g l c P 1$ promoter, showed only $30 \%$ glucose utilization at $96 \mathrm{~h}$ (not shown). The glucose consumption rates correlate well with the previously observed growth rates for the different strains (Fig. 6b).

It is interesting that $S$. clavuligerus::pRA-AB-K shows good growth in glucose but is not able to use lactose, sucrose, mannitol, galactose or fructose $(1 \%)$ as sole carbon source, confirming that GlcP1 is a glucose-specific transporter (see Discussion).

The expression of the heterologous $\mathrm{glcP} 1$ gene from its own promoter (in S. clavuligerus::pRA-AB) and from the $g l c P$ promoter (in S. clavuligerus::pRA-CB, which does not grow on glucose) was compared using the glcP1-specific oligonucleotides GLCP1/GLCP2. As observed in Fig. 6(c), no amplification of the $g l c P 1$ transcript occurred in the negative control S. clavuligerus::pRA (lane 1) due to the specificity of the primers. In $S$. clavuligerus::pRA-AB (lanes 3), a clear signal was observed for $g l c P 1$ that was slightly less intense than that observed for $S$. coelicolor M145 (lane 2). Amplification of the glcP1 transcript in S. clavuligerus::pRA-CB (lanes 4) was very weak when compared with that of $S$. clavuligerus:: pRA-AB (lanes 3), confirming the low efficiency of the $g l c P$ promoter, but was still perceptible when compared with the negative control S. clavuligerus::pRA (lane 1). In this figure it can be seen that glucose does not stimulate transcription of the $g l c P 1$ gene.

In summary, the lack of glucose utilization in S. clavuligerus can be complemented using the $g l c P 1$ gene of $S$. coelicolor or, simply, the glcP1 promoter coupled to the $S$. clavuligerus promoterless $g l c P$ gene.

\section{DISCUSSION}

Glucose utilization in Streptomyces species depends on two key proteins, a glucose permease and a glucose kinase. S. coelicolor glucose kinase formation is constitutive, but its activation is mediated by a glucose-dependent posttranslational modification (van Wezel et al., 2007). S. clavuligerus contains a constitutively expressed glkA gene, and shows glucose kinase activity $25 \%$ lower than the activity found in S. coelicolor. Higher levels of glucose kinase activity, obtained by expression of the S. coelicolor glkA gene in $S$. clavuligerus, did not confer the ability to grow on glucose upon the $S$. clavuligerus transformants. The same results were obtained by increasing the copy number of orf 3 , the homologous gene to $S$. coelicolor SCO2127. This gene has been reported to stimulate 
(a)
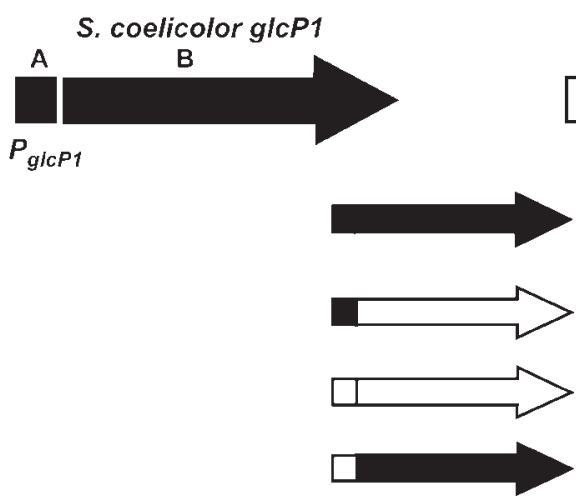

pRA-AD

PRA-CD

pRA-CB

\section{(b)}
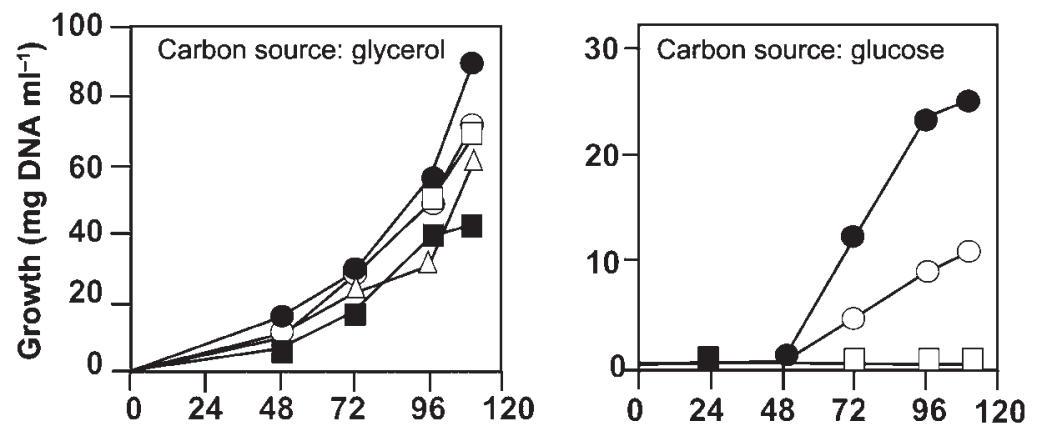

Time (h)
Fig. 5. Assembly of $S$. clavuligerus and $S$. coelicolor promoters and glucose permease genes. (a) The g/cP1 gene of $S$. coelicolor and the $g / c P$ gene of $S$. clavuligerus with their promoters are shown above. The different promoters and genes assembled in plasmid pRA give the constructs pRA-AB, pRA-AD, pRA-CD and pRA-CB, shown below. (b) Growth of $S$. clavuligerus::pRA $(\triangle)$, S. clavuligerus: : pRA-AB (๑), S. clavuligerus:: pRA-AD (O), S. clavuligerus::pRA-CD ( $\square$ ) and $S$. clavuligerus::pRA-CB ( $\square$ ) on BM medium supplemented with either $1 \%(\mathrm{v} / \mathrm{v})$ glycerol (left panel) or glucose (right panel). transcription of the glucokinase gene in Streptomyces peucetius (Guzmán et al., 2005).

S. coelicolor has two genes, $g l c P 1$ and $g l c P 2$ (probably originating by gene duplication), to transport glucose (van Wezel et al., 2005). Expression of $g l c P 1$ is essential for growth on glucose, while $g l c P 2$ can be deleted without appreciable effects (van Wezel et al., 2005). S. clavuligerus, like several other Streptomyces species, contains a single glucose permease gene $(g l c P)$, encoding a protein similar to those of S. coelicolor ( $80 \%$ identical). The glcP2 promoter region, as well as the $S$. clavuligerus $g l c P$ promoter, differ significantly from those of $g l c P 1$ of $S$. coelicolor and the homologous $S$. avermitilis araE gene.

In this work we demonstrate that the native $S$. clavuligerus $g l c P$ promoter has very low expression. The residual transcription level, originating from the $g l c P$ promoter, is not sufficient for glucose utilization. A similar situation occurs with the $S$. coelicolor glcP2 promoter. This gene $(g l c P 2)$ is able to confer glucose utilization upon $S$. clavuligerus when expressed from the tetracycline-induced tcp830 promoter (results not shown), indicating that the lack of function of $g l c P 2$ in $S$. coelicolor is, again, due to poor expression from an inefficient promoter. The similarity with the lack of expression of the second glucose permease gene of $S$. coelicolor further supports this hypothesis. It is likely that the redundancy of identical $g l c P 1$ and $g l c P 2$ genes in $S$. coelicolor has favoured the decay of expression of $g l c P 2$ in this actinomycete.
S. clavuligerus glucose permease is functional, as shown by the growth on glucose of $S$. clavuligerus::pRA-AD. This was confirmed by complementation of $E$. coli glucose transport-negative mutants with $g l c P$ expressed from the lacZ promoter.

Carbon catabolite regulation in S. coelicolor, in contrast to the regulation mechanism in enterobacteria (Görke \& Stülke, 2008), appears to be exerted through the constitutive glucose kinase (GlkA) (Angell et al., 1992, 1994). Proteins GlkA and GlcP have been proposed to form an active complex, GlkA-GlcP, to internalize and phosphorylate glucose (van Wezel et al., 2007). Our results support this proposal. The best growth of $S$. clavuligerus on glucose was obtained when the homologous $g l c P 1$ and $g l k A$ genes, both from S. coelicolor, were introduced into S. clavuligerus; however, when one glucose permease of $S$. coelicolor was combined with the glucose kinase of $S$. clavuligerus the growth and glucose utilization rate were lower.

S. clavuligerus appears to have adapted to grow on glycerol, maltose or even amino acids as carbon sources, losing the ability to utilize other carbon sources. Introduction of the S. coelicolor glcP1 gene results in utilization of glucose but not of other hexoses. This provides conclusive evidence that $g l c P$, the direct orthologue of $g l c P 1$ of $S$. coelicolor, indeed encodes the glucose-specific transporter of S. clavuligerus. The lack of utilization of several other sugars in $S$. clavuligerus suggests that this actinomycete is defective in several sugar transporters. Even if the genes 
(a)

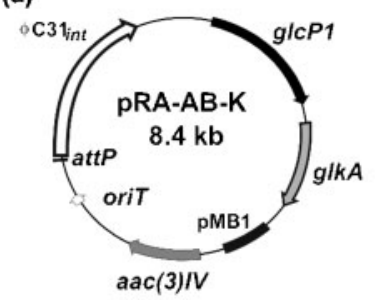

(b)

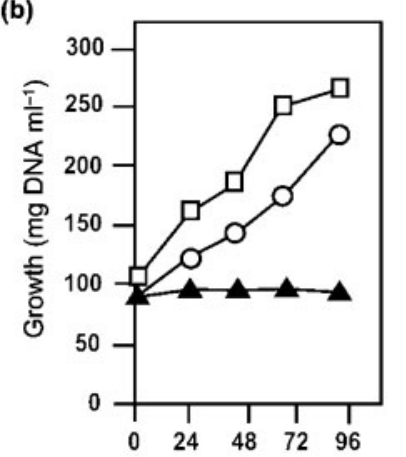

(c)
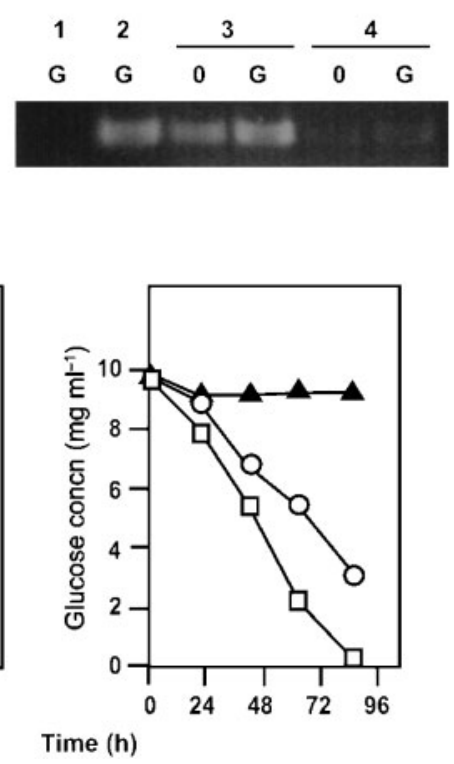

Fig. 6. Effect of $S$. coelicolor $g / c P 1$ and $g / k A$ genes on the growth of $S$. clavuligerus. (a) Plasmid pRA-AB-K used to introduce glkAglcP1 in S. clavuligerus. (b) Growth of $S$. clavuligerus : : pRA ( $\mathbf{A})$, S. clavuligerus:: $\mathrm{pRA}-\mathrm{AB}-\mathrm{K}(\square)$ and $S$. clavuligerus::pRA-AB $(\bigcirc)$ in glucose-supplemented BM medium (left panel), and glucose utilization by the same $S$. clavuligerus exconjugants (right panel). (c) Heterologous expression of $\mathrm{g} / \mathrm{c} P 1$ controlled by either the $S$. coelicolor g/cP1 or the S. clavuligerus g/cP promoter, tested by RT-PCR. RNA obtained in SA medium at 0 and $9 \mathrm{~h}(\mathrm{G})$ after glucose supplementation. (1) $S$. clavuligerus::pRA, (2) $S$. coelicolor, (3) S. clavuligerus: : pRA-AB, (4) S. clavuligerus : : pRA-CB.

encoding those transporters are expressed, lack of interaction of the modified proteins with other proteins in the membrane or in the cytoplasm may explain the lack of growth on those carbon sources.

\section{ACKNOWLEDGEMENTS}

This work was supported by grants from the Spanish Ministry of Science and Technology (BIO2003-03274, BIO2006-14853) and the European Community (Actinogen LSHMCT-2004-005224). DNA sequences were provided via Dr Wilbert Heijne (DSM, The Netherlands). Plasmids pFT76 and pFT256 were provided by Dr Fritz Titgemeyer (FriedrichAlexander-Universität, Erlangen, Germany), and the glucose transportdeficient mutants E. coli LM1 and E. coli LR2-175 by Dr Knut Jahreis (Osnabrück University, Germany).

\section{REFERENCES}

Aharonowitz, Y. \& Demain, A. L. (1978). Carbon catabolite regulation of cephalosporin production in Streptomyces clavuligerus. Antimicrob Agents Chemother 14, 159-164.

Aidoo, K. A., Wong, A., Alexander, D. C., Rittammer, R. A. \& Jensen, S. E. (1994). Cloning, sequencing and disruption of a gene from

Streptomyces clavuligerus involved in clavulanic acid biosynthesis. Gene 147, 41-46.

Angell, S., Schwarz, E. \& Bibb, M. J. (1992). The glucose kinase gene of Streptomyces coelicolor A3(2): its nucleotide sequence, transcriptional analysis and role in glucose repression. Mol Microbiol 6, 2833-2844.

Angell, S., Lewis, C. G., Buttner, M. J. \& Bibb, M. J. (1994). Glucose repression in Streptomyces coelicolor A3(2): a likely regulatory role for glucose kinase. Mol Gen Genet 244, 135-143.

Aulkemeyer, P., Ebner, R., Heilenmann, G., Jahreis, K., Schmid, K., Wrieden, S. \& Lengeler, J. W. (1991). Molecular analysis of two fructokinases involved in sucrose metabolism of enteric bacteria. Mol Microbiol 5, 2913-2922.

Bertram, R., Schlicht, M., Mahr, K., Nothaft, H., Saier, M. H., Jr \& Titgemeyer, F. (2004). In silico and transcriptional analysis of carbohydrate uptake systems of Streptomyces coelicolor A3(2). J Bacteriol 186, 1362-1373.

Burton, K. (1968). Determination of DNA concentration with diphenylamine. Methods Enzymol 12, 163-166.

Chatterjee, S. \& Vining, L. C. (1981). Nutrient utilization in actinomycetes. Induction of $\alpha$-glucosidases in Streptomyces venezuelae. Can J Microbiol 27, 639-645.

Garcia-Domínguez, M., Martín, J. F. \& Liras, P. (1989). Characterization of sugar uptake in wild-type Streptomyces clavuligerus, which is impaired in glucose uptake, and in a glucose-utilizing mutant. J Bacteriol 171, 6808-6814.

Görke, B. \& Stülke, J. (2008). Carbon catabolite repression in bacteria: many ways to make the most out of nutrients. Nat Rev Microbiol 6, 613-624.

Gust, B., Challis, G. L., Fowler, K., Kieser, T. \& Chater, K. F. (2003). PCR-targeted Streptomyces gene replacement identifies a protein domain needed for biosynthesis of the sesquiterpene soil odor geosmin. Proc Natl Acad Sci U S A 100, 1541-1546.

Guzmán, S., Carmona, A., Escalante, L., Imriskova, I., López, R., Rodríguez-Sanoja, R., Ruiz, B., Servín-González, L., Sánchez, S. \& Langley, E. (2005). Pleiotropic effect of the SCO2127 gene on the glucose uptake, glucose kinase activity and carbon catabolite repression in Streptomyces peucetius var. caesius. Microbiology 151, 1717-1723.

Hanahan, D. (1983). Studies on transformation of Escherichia coli with plasmids. J Mol Biol 166, 557-580.

Hindle, Z. \& Smith, C. P. (1994). Substrate induction and catabolite repression of the Streptomyces coelicolor glycerol operon are mediated through the GylR protein. Mol Microbiol 12, 737-745.

Kieser, T., Bibb, M. J., Buttner, M. J., Chater, K. F. \& Hopwood, D. A. (2000). Practical Streptomyces genetics. Norwich, UK: John Innes Foundation.

Lengeler, J., Auburger, A. M., Mayer, R. \& Pecher, A. (1981). The phosphoenolpyruvate-dependent carbohydrate: phosphotransferase system enzymes II as chemoreceptors in chemotaxis of Escherichia coli K 12. Mol Gen Genet 183, 163-170.

MacNeil, D. J., Occi, J. L., Gewain, K. M., MacNeil, T., Gibbons, P. H., Ruby, C. L. \& Danis, S. J. (1992). Complex organization of the Streptomyces avermitilis genes encoding the avermectin polyketide synthase. Gene 115, 119-125.

Mesak, L. R., Mesak, F. M. \& Dahl, M. K. (2004). Bacillus subtilis GlcK activity requires cysteines within a motif that discriminates microbial glucokinases into two lineages. BMC Microbiol 4, 6-15.

Nothaft, H., Dresel, D., Willimek, A., Mahr, K., Niederweis, M. \& Titgemeyer, F. (2003a). The phosphotransferase system of Streptomyces coelicolor is biased for $\mathrm{N}$-acetylglucosamine metabolism. J Bacteriol 185, 7019-7023. 
Nothaft, H., Parche, S., Kamionka, A. \& Titgemeyer, F. (2003b). In vivo analysis of HPr reveals a fructose-specific phosphotransferase system that confers high-affinity uptake in Streptomyces coelicolor. J Bacteriol 185, 929-937.

Paget, M. S., Chamberlin, L., Atrih, A., Foster, S. J. \& Buttner, M. J. (1999). Evidence that the extracytoplasmic function sigma factor $\sigma^{\mathrm{E}}$ is required for normal cell wall structure in Streptomyces coelicolor A3(2). J Bacteriol 181, 204-211.

Rigali, S., Nothaft, H., Noens, E. E., Schlicht, M., Colson, S., Müller, M., Joris, B., Koerten, H. K., Hopwood, D. A. \& other authors (2006). The sugar phosphotransferase system of $S$. coelicolor is regulated by the GntR-family regulator DasR and links $\mathrm{N}$-acetylglucosamine metabolism to the control of development. Mol Microbiol 61, 1237-1251.

Rigali, S., Titgemeyer, F., Barends, S., Mulder, S., Tomae, A. W., Hopwood, D. A. \& van Wezel, G. P. (2008). Feast or famine: the global regulator DasR links nutrient stress to antibiotic production by Streptomyces. EMBO Rep 9, 670-675.
Rodríguez-García, A., Barreiro, C., Santos-Beneit, F., Sola-Landa, A. \& Martín, J. F. (2007). Genome-wide transcriptomic and proteomic analysis of the primary response to phosphate limitation in Streptomyces coelicolor M145 and in a $\Delta$ phoP mutant. Proteomics 7, 2410-2429.

Sambrook, J., Fritsch, E. F. \& Maniatis, T. (1989). Molecular Cloning: a Laboratory Manual, 2nd edn. Cold Spring Harbor, NY: Cold Spring Harbor Laboratory.

van Wezel, G. P., Mahr, K., König, M., Traag, A., Pimentel-Schmitt, E. F., Willimek, A. \& Titgemeyer, F. (2005). GlcP constitutes the major glucose uptake system of Streptomyces coelicolor A3(2). Mol Microbiol 55, 624-636.

van Wezel, G. P., König, M., Mahr, K., Nothaft, H., Thomae, A. W., Bibb, M. \& Titgemeyer, F. (2007). A new piece of an old jigsaw: glucose kinase is activated posttranslationally in a glucose transportdependent manner in Streptomyces coelicolor A3(2). J Mol Microbiol Biotechnol 12, 67-74.

Edited by: M. Paget 